\title{
Analysis of Gas Leakage and Diffusion Model
}

\author{
He Hong 1, a, Chen Wen-haor 1,2,b, Liu Mei, ${ }^{2, \mathrm{c}}$ and Xu Xiao-ling²
}

${ }^{1}$ Tianjin Key Laboratory for Control Theory \& Applications in Complicated Systems, School of Electrical Engineeing and Automation, Tianjin University of Technology, Tianjin 300384, China;

${ }^{2}$ Guangdong University of Petrochemical Technology, Maoming 525000,China; aheho604300@126.com, b598393356@qq.com, c879643579@qq.com

Keywords: Gaussian Plume Model,Gaussian Puff Model, Gas turbulence model, MATLAB. Abstract. Gas diffusion model can provide important analytical data in the detection of toxic gases., and the commonly used gas diffusion model has Gaussian plume model, Gaussian puff model and gas turbulence model. In this paper according to actual atmospheric environment of Sinopec Maoming Branch of ethylene plant in Maoming, of chlorine leakage accident simulation. Through the MATLAB software, three kinds of gas diffusion model were simulated and the data were compared and analyzed through the experiments, and the gas concentration data with practical reference value is obtained.

\section{Introduction}

Chlorine gas is a kind of poisonous gas, and it is also one of the main chemical raw materials. According to statistics, chlorine gas is one of the highest chemical accidents in the chemical industry in our country. Chlorine leakage and explosion accidents usually cause the people and the production staff of poisoning casualties, bringing serious impact on people's lives and property security. Therefore, establishing chlorine gas leakage and diffusion model, making reasonable simulation of the diffusion of chlorine gas leakage and analysis of the impact of chlorine diffusion and dangerous area so as to provide a scientific basis for the accident emergency treatment and the risk management of toxic and hazardous substances. At present, there are lots of known gas diffusion models, the gas diffusion model can be divided into two categories: static model and dynamic model. we mainly study the Gauss model and the gas turbulence model in the static model in this paper. Gauss model and gas turbulence model are often used in the study of point source diffusion. In this paper, the diffusion motion of chlorine gas leakage is simulated based on the two models, and carry out comparative analysis.

\section{Gauss model and gas turbulence model}

\section{Gauss model}

The Gauss model includes two types: the Gauss plume model and the Gaussian puff model, the smoke plume model is suitable for continuous point source diffusion, which is mainly used for the case that the source is continuous source or discharge time is greater than or equal to the diffusion time, and the smoke cluster model is suitable for the diffusion of instantaneous leakage.

1) Gauss Plume Model

The Gauss plume model can be used to simulate the concentration distribution of the pollutant in the air along the lower direction. The model satisfies the formula (2-1) under the condition that the atmospheric stability, wind direction and wind speed are not changed with time:

$$
c(x, y, z)=\frac{Q}{2 \pi \sigma_{y} \sigma_{z} u} \exp \left(-\frac{y^{2}}{2 \sigma_{y}^{2}}\right)\left\{\exp \left[\frac{(z-H)^{2}}{2 \sigma_{z}^{2}}\right]+\exp \left[\frac{(z+H)^{2}}{2 \sigma_{z}^{2}}\right]\right\}
$$

In the formula: $\mathrm{c}(\mathrm{x}, \mathrm{y}, \mathrm{z})$ - gas concentration of some downwind point $\mathrm{c}(\mathrm{x}, \mathrm{y}, \mathrm{z}), \mathrm{mg} / \mathrm{m}^{3} ; \mathrm{Q}-\mathrm{gas}$ source strength, $\mathrm{mg} / \mathrm{s} ; \mathrm{H}$ - effective source height,, $\mathrm{m} ; \mathrm{u}$-average wind speed, $\mathrm{m} / \mathrm{s} ;, \sigma_{y}, \sigma_{z}-$ diffusion parameters of the direction of $\mathrm{y}$ and $\mathrm{z}, \mathrm{m} ;{ }^{\sigma_{y}}, \sigma_{z}$ are under the influence of environmental 
stability, which is divided into A F 6 grades according to the wind speed, A has the weakest stability, F the most stable.

2)Gaussian Puff Model

The Gaussian puff model is suitable for a long period of time of release or short time of sudden release or a large number of toxic and harmful gases, that is the discharge time is shorter than the diffusion time, so the calculation of ground concentration should be based on the smoke cluster model at this time:

$$
c(x, y, z)=\frac{2 Q}{(2 \pi)^{3 / 2} \sigma_{x} \sigma_{y} \sigma_{z}} \exp \left(-\frac{x^{2}}{2 \sigma_{x}^{2}}\right) \exp \left(-\frac{y^{2}}{2 \sigma_{y}^{2}}\right) \exp \left(-\frac{z^{2}}{2 \sigma_{z}^{2}}\right)
$$

In the formula, the center of the smoke cluster is at the origin. Other parameters significance as Gauss plume model section shows.

In general, the leakage mode is a continuous discharge in a finite time, and in the period of the continuous emission source can be regarded as a transient puff to one point in the leeward (x, y, z) concentration superposition, obtaining the following diffusion model :

$$
\left\{\begin{array}{l}
c=\sum_{i=1}^{M} c_{i}\left(x, y, z, t-t_{i}\right) \\
\left.c_{i}(x, y, z)=\frac{2 Q}{(2 \pi)^{3 / 2} \sigma_{x} \sigma_{y} \sigma_{z}} \exp -\frac{(x-u t)^{2}}{2 \sigma_{x}^{2}}-\frac{y^{2}}{2 \sigma_{y}^{2}}-\frac{H^{2}}{2 \sigma_{z}^{2}}\right)
\end{array}\right.
$$

In the formula,; $\mathrm{u}$-average wind speed, $\mathrm{m} / \mathrm{s}, \mathrm{M}$ is the number of cigarettes 。

\section{Gas turbulence model}

Assumes ${ }_{c\left(\overrightarrow{\mathbf{r}}, \overrightarrow{\mathbf{r}}_{0}, t\right)}$ represents the gas concentration in $\overrightarrow{\boldsymbol{r}}(x, y, z)$ at the moment $\mathrm{t}$, the unit is $\mathrm{mg} / \mathrm{m}^{3}$, $\overrightarrow{r_{0}}=\left(x_{0}, y_{0}, z_{0}\right)$ represents the source location. When the wind exists, and gas began to spread the initial time is $\mathrm{t}_{0}$ 。

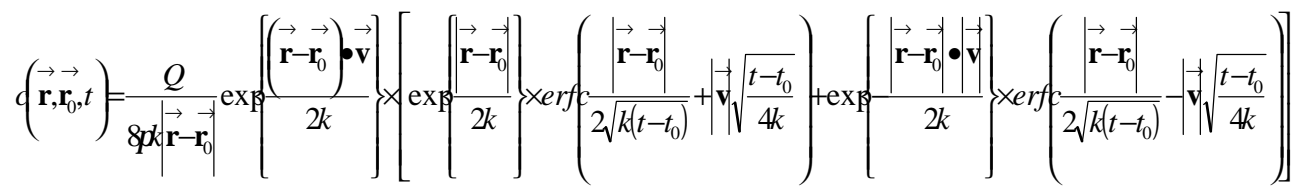

By formula (2-4), we can derive the formula under the windless condition.

$$
c(\overrightarrow{\boldsymbol{r}}, t)=\frac{Q}{4 \pi k\left|\overrightarrow{\boldsymbol{r}}-\overrightarrow{\boldsymbol{r}}_{0}\right|} \operatorname{erfc}\left(\frac{\left|\overrightarrow{\boldsymbol{r}}-\overrightarrow{\boldsymbol{r}}_{0}\right|}{2 \sqrt{k\left(t-t_{0}\right)}}\right)
$$

\section{Simulation results of the chlorine leak and diffusion model analysis}

Chlorine leakage accident occurred in this study Maoming Petrochemical Yixi plant for the study .Therefore, the diffusion coefficient of the urban in Table 1:

Table 1 Diffusion coefficients under different atmospheric stabilty

\begin{tabular}{ccc}
\hline Pascual category & $\sigma_{y} /(m)$ & $\sigma_{z} /(m)$ \\
\hline A B & $0.32 x(1+0.0004 x)^{-\frac{1}{2}}$ & $0.24 x(1+0.0001 x)^{-\frac{1}{2}}$ \\
C & $0.22 x(1+0.0004 x)^{-\frac{1}{2}}$ & $0.2 x$ \\
D & $0.16 x(1+0.0004 x)^{-\frac{1}{2}}$ & $0.14 x(1+0.0003 x)^{-\frac{1}{2}}$ \\
E $\sim \mathrm{F}$ & $0.11 x(1+0.0004 x)^{-\frac{1}{2}}$ & $0.08 x(1+0.0015 x)^{-\frac{1}{2}}$
\end{tabular}

According to "Technical Guidelines for Environmental Assessment - Atmospheric Environment", Table 1, atmospheric stability and atmosphere of sunshine, wind speed and cloud related. Based on five categories of surface wind speed, three types of solar radiation during the day and two night cloudiness, Pascual make weather diffusion into the unstable (A), unstable (B), weakly unstable (C), neutral (D), more stable (E) and stable (F) and other six categories. 
We hypothesized that in at 15:00 on December 1 chlorine leak occurred in Hexi petrochemical plant, then westerly winds of grade 1 , with an average wind speed of $1 \mathrm{~m} / \mathrm{s}$, sunny weather, skies cloud about $1 / 3$. Out of control due to the pressure relief valve, chlorine 1000kg moment all leaks.

\section{Simulation Result}

According to the meteorological data in the area of the enterprise, we can know that the atmospheric stability of the area is B.The chlorine tank of the enterprise is placed on the ground, so the leakage of the storage tank is the point source of the ground, assuming instantaneous leakage, the leakage of the gas according to the Gauss diffusion model of the ground instantaneous point source diffusion model for analysis. Through the MATLAB software model for numerical simulation, Gaussian plume model simulation results as shown in Figure 1 Figure 2 shown in Figure 1 shows the $\mathrm{z}=0$ for the Gaussian plume model simulation results, figure 2 shows the Gaussian plume model simulation of the three dimensional graphics. While the Gaussian puff model simulation results, as shown in Figure $2 \sim$ Figure 6 shows , 3,5, respectively is the plan of $t=60 \mathrm{~s} t=150$ s. Figure 4,6 is three dimensional graphics; gas turbulence model simulation results as shown in Fig. $7 \sim 8$, shown in Figure 7 is $Z=0$ when the gas turbulence model simulation results, shown in Figure 8 is gas turbulence simulation model for 3D graphics.

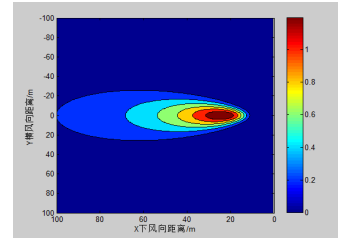

Fig. 1

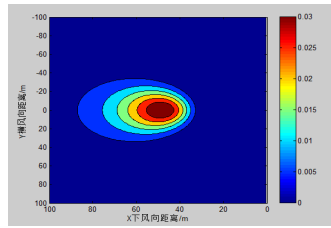

Fig. 5

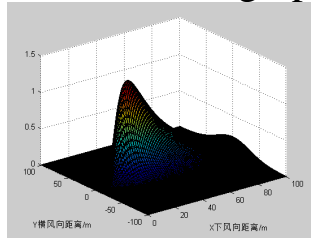

Fig. 2

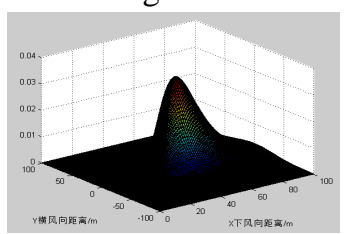

Fig. 6

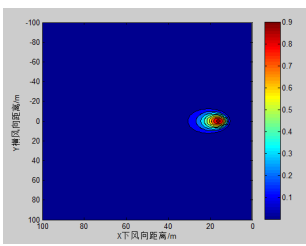

Fig. 3

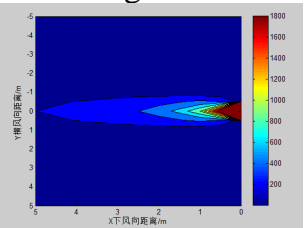

Fig. 7

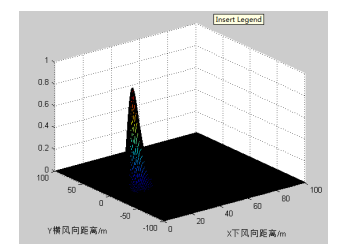

Fig. 4

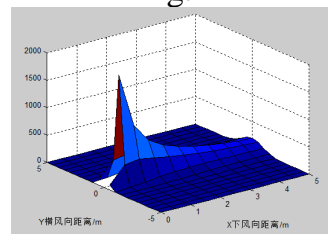

Fig. 8

\section{For the simulation results of correlation analysis}

We have randomly selected three points are calculated Based on different models(A(91.5736, 99.6135, 17.0708)、B(25.7508, 5.9780, 29.6676)、C(49.8364, 48.9253, 62.4060)), The calculated result is displayed in the following table:

Table 2 Calculation Data of Random Coordinate Point

\begin{tabular}{ccccc}
\hline & $\begin{array}{c}\text { Gaussian plume } \\
\text { model } \\
\left(\mathrm{mg} / \mathrm{m}^{3}\right)\end{array}$ & $\begin{array}{c}\text { Gaussian puff } \\
\text { model } \\
(60 \mathrm{~s})\left(\mathrm{mg} / \mathrm{m}^{3}\right)\end{array}$ & $\begin{array}{c}\text { Gaussian puff } \\
\text { model } \\
(150 \mathrm{~s})\left(\mathrm{mg} / \mathrm{m}^{3}\right)\end{array}$ & $\begin{array}{c}\text { Gas turbulence } \\
\text { model } \\
\left(\mathrm{mg} / \mathrm{m}^{3}\right)\end{array}$ \\
\hline $\mathrm{A}$ & 0.334 & 0 & 0.285 & 0 \\
$\mathrm{~B}$ & 10.408 & 8.347 & 5.859 & 6.208 \\
$\mathrm{C}$ & 4.154 & 3.583 & 2.563 & 5.467 \\
\hline
\end{tabular}

The numerical value of the above table shows that at the point of $A$, the calculated values of the Gauss puff model and the gas turbulence model are quite close, the calculated value of the Gauss plume model is much higher than that of the other two models. At the point of $\mathrm{B}$, the calculated values of Gauss plume model and the Gauss puff model at 60s are similar, and the calculated values of the Gas turbulence model and the Gauss puff model are similar at 150s; At the point of $\mathrm{C}$, the calculated value of the Gas turbulence model is higher than that of the Gauss model, and the calculated values of the Gauss plume model and the Gauss puff model are similar.

From the simulation chart above, we can find that the simulation results of Gauss plume model are similar to that of Gauss puff model, and the difference lies in that the Gauss puff model takes the movement of the gas source point into account; The simulation results of the Gas turbulence model and the Gauss model are obviously different, and the Gauss model simulation shows that the shape of gas diffusion is elliptical, and the simulation diagram of Gas turbulence model shows that the gas diffusion shape is cone shape. 


\section{Test of chlorine concentration}

\section{Equipment}

Experiments using CVT-IOT-VS experimental box, which (CVT-IOT-VS) networking comprehensive experiment teaching system includes a wireless chip display gateway module , gateway directly by TI company of CC2530 wireless microcontroller as the core processor, the wireless sensor information can be transferred to the PC monitoring software in the test, In addition, the main experimental environment is a box of 1 cubic meters of transparent glass , 1 bottles of net weight $100 \mathrm{~g}$ of chlorine, a chlorine sensor module and adjustable air blower; In the experiment, In a glass case inside a thin line to three chlorine sensor module in ABC three-point coordinates, remove the pre-prepared chlorine, placed in the center of the glass box $(\sqrt{2} / 2, \sqrt{2} / 2,0)$, then create mechanisms westerly $1 \mathrm{~m} / \mathrm{s}$ wind speed, spread of detecting chlorine group. And then test whether the sensor can correctly detect the concentration of chlorine.

\section{Result}

Through the above experiments, the chlorine concentration of A B C in 60 s and 150s time were detected respectively. The results of the experiment are as follows.

Table 3 Experiment To Detect The Concentration of Selected Results

\begin{tabular}{cccc}
\hline Time & $\mathrm{A}$ & $\mathrm{B}$ & $\mathrm{C}$ \\
\hline $60(\mathrm{~s})$ & 0 & 7.847 & 4.823 \\
$150(\mathrm{~s})$ & 0.364 & 4.442 & 2.687
\end{tabular}

\section{Conclusion}

Combined with the experimental data, we can get the difference between the experimental data and the model data by calculation. Shown in Table 4:

Tab. 4 Calculation Results of Model And Error of Experimental Results

\begin{tabular}{lllll}
\hline Time (s) & $\begin{array}{l}\text { Selected } \\
\text { point }\end{array}$ & Gaussian plume model & Gaussian puff model & Gas turbulence model \\
\hline \multirow{3}{*}{$60 \mathrm{~s}$} & A & $33.4 \%$ & 0 & 0 \\
& $\mathrm{~B}$ & $22.6 \%$ & $5.9 \%$ & $26.4 \%$ \\
& $\mathrm{C}$ & $16.1 \%$ & $34.6 \%$ & $11.7 \%$ \\
$150 \mathrm{~s}$ & $\mathrm{~A}$ & $8.9 \%$ & $27.7 \%$ & $36.4 \%$ \\
& $\mathrm{~B}$ & $57.3 \%$ & $24.1 \%$ & $28.4 \%$ \\
& $\mathrm{C}$ & $35.3 \%$ & $4.8 \%$ & $50.8 \%$ \\
\hline
\end{tabular}

According to table 4 , in 60 s time, the average error of the Gauss smoke model is $13.5 \%$, the average error of the Gauss plume model is $24 \%$, and the average error of the Gas turbulence model is $12.7 \%$; At 150 s time, the average error of the Gauss model is $18.8 \%$, the average error of the Gauss plume model is $33.8 \%$, and the average error of the Gas turbulence model is $38.5 \%$. It can be seen that the Gauss puff model is more reliable than the Gauss plume model and the Gas turbulence model, and the simulation results are more close to the actual situation.

In addition, it can be seen from the simulation in this paper, the gas diffusion movement of the Gauss smoke cluster model is more diversified and close to the actual situation. Therefore, it is concluded that the Gauss puff model is more suitable for the calculation and Simulation of gas leakage diffusion than the Gauss plume model and the Gas turbulence model. 


\section{Acknowledgements}

Fund Project: Tianjin Science and Technology Special Fund to support major science and technology projects (14ZCDGSF00028), "Tianjin higher education institution Innovation Team Training Program funded" Project No. TD12-5015

\section{References}

[1]Zhou De Hong, Wang Liu Jiu. chlorine leak diffusion risk analysis. [J]. Wuhan Institute of Technology, 2011, 33(6):76-81.

[2]Ye Dong Fen, Ye Qiao Long, Luo Wei Chen. Based on calculation and dangerous chemical spill area Gaussian Model [J]. Computers and Applied Chemistry, 2012,29(2):195-199.

[3]N.I. Drobyshevsky, L.I. Zaichik, R.V. Mukin, V.F. Strizhov, and A.S. Filippov.Development and application of a diffusion-inertia model for simulating gas-dispersed turbulent flows [J]. Nuclear Safety Institute RAS,2009,16(4):521-538.

[4]Li Yun Yun. Improved Gaussian plume model and its application in hazardous chemical spill simulation [D]. Guangzhou: Guangzhou University, 2013.

[5]Li Bing Jing, Tong Ji Long, Pan Feng, etc. Gaussian puff model in petrochemical projects Environmental Risk Assessment atmosphere [J]. Environmental Engineering, 2013,31(3):139-143. 